\title{
Acute Respiratory Distress Syndrome Due to Vivax Malaria: Case Report and Literature Review
}

\author{
André V. Lomar ${ }^{1,2}$, José E. Vidal2,3, \\ Frederico P. Lomar ${ }^{1}$, Carmen Valente Barbas ${ }^{1}$, \\ Gustavo Janot de Matos $^{1}$ and Marcos Boulos ${ }^{3}$
}

\author{
Teaching and Research Israelit Institute Albert Einstein, Albert \\ Einstein Hospital ${ }^{1}$, Emílio Ribas Institute ${ }^{2}$, School of Medicine - \\ University of São Paulo ; São Paulo, SP, Brazil
}

\begin{abstract}
Severe pulmonary involvement in malaria has been frequently reported in cases of Plasmodium falciparum infection, but rarely in vivax malaria. Among the 11 previous cases of vivax-related severe respiratory involvement described in the literature, all except one developed it after the beginning of anti-malarial treatment; these appear to correspond to an exacerbation of the inflammatory response. We report the case of a 43-year-old Brazilian woman living in a malariaendemic area, who presented acute respiratory distress syndrome (ARDS) caused by $P$. vivax before starting anti-malarial treatment. The diagnosis was made based on microscopic methods. A negative rapid immunochromatographic assay, based on the detection of Histidine Rich Protein$\mathbf{2}$ (HRP-2) of $\boldsymbol{P}$. falciparum, indicated that falciparum malaria was unlikely. After specific antiplasmodial therapy and intensive supportive care, the patient was discharged from the hospital. We conclude that vivax malaria-associated ARDS can develop before anti-malarial therapy.

Kev Words: Malaria, Plasmodium vivax, acute respiratory distress syndrome, acute respiratory failure, Brazil.
\end{abstract}

Malaria is an increasingly common public health problem in many parts of the world [1,2]. The increase in the number of cases in endemic areas, human migration, and increased world travel have led to many cases of imported malaria in countries where it had been eradicated, making it a global health problem [3,4].Classically, severe malaria is caused by Plasmodium falciparum; this disease is manifested by severe anemia, renal failure, acute respiratory failure, hypoglycemia, shock, and/or central nervous system involvement [5]. Plasmodium vivax is endemic in many countries of Asia and South America, and it usually presents as a benign febrile acute disease. We report a rare case of acute respiratory distress syndrome (ARDS) caused by P. vivax.

Received on 3 July 2005; revised 11 August 2005.

Address for correspondence: Dr. André V. Lomar. Hospital Albert Einstein, Av. Albert Einstein 627, sala 1314, São Paulo, SP. Zip code: 05651-901, Brazil. Telephone: 55-11-37425467. Fax: 55-11-37473534.E-mail: andre@lomar.med.br

The Brazilian Journal of Infectious Diseases 2005;9(5):425-430 (C) 2005 by The Brazilian Journal of Infectious Diseases and Contexto Publishing. All rights reserved.

\section{Case Report}

A 43-year-old woman was admitted to the Albert Einstein Hospital, Sao Paulo, Brazil, because of fever and prostration. She had been well until three days earlier, when fever, myalgia, headache and fatigue developed. On the day of admission, the patient complained of worsening of symptoms. She was fully oriented, alert, and able to talk coherently.

She lived in Manaus (the capital of Amazonia State in Brazil - in the middle of the Amazon forest), but frequently traveled to Santos (a city located on the coast of Sao Paulo State). When in Manaus, she remained within the city limits and had not visited the Amazon forest. The patient had no history of previous malaria, and she had never received anti-malarial prophylaxis.

The temperature was $37.9^{\circ} \mathrm{C}$, the blood pressure was $81 / 64 \mathrm{~mm} \mathrm{Hg}$, and the pulse was 115 beats per minute. The oxygen saturation was $94 \%$, while the patient was breathing room air. Pulmonary and cardiac sounds were normal. The liver was palpable at the costal 
margin. The rest of the examination was unremarkable. Relevant laboratory investigations included the following: hemoglobin level $=12.4 \mathrm{~g} / \mathrm{dL}$, hematocrit $=$ $37.2 \%$, white blood cell count $=3,400 / \mathrm{mL}$, with $92 \%$ neutrophils, $6 \%$ lymphocytes, and $2 \%$ monocytes. The platelet count was $65,000 / \mathrm{mm}^{3}$, and the serum creatinine level was $0.7 \mathrm{mg} / \mathrm{dL}$. Prothrombin and activated partial tissue thromboplastin times were normal, as were the results of tests for serum electrolytes, and serum liver enzymes. Abdominal ultrasonography and chest X-ray were normal.

Forty-eight hours after hospital admission, the patient experienced dry cough, tachypnea, dyspnea, and hypoxemia $(82 \%)$, as assessed by oxygen desaturation on pulse oximetry. She had not received intravenous infusion and she had not presented clinical manifestations of fluid overload. Oxygen (Venturi mask $40 \%$ ) was delivered to the patient, and a chest X-ray and computed tomography of the lungs were performed (Figures 1 and 2).

The patient was sent to the Intensive Care Unit, where invasive mechanical ventilation was initiated. Her $\mathrm{FiO}_{2} / \mathrm{PaO}_{2}$ ratio was $<200$, consistent with a diagnosis of ARDS. The blood pressure was $80 / 45 \mathrm{mmHg}$ and noradrenaline was initiated. Ceftriaxone, initiated at admission, was stopped and Cefepime $2 \mathrm{~g}$ bid IV and Levofloxacin $500 \mathrm{mg}$ qd IV were initiated. The echocardiogram showed normal cardiac function. Due to severe thrombocytopenia $\left(17,000 / \mathrm{mm}^{3}\right)$, no pulmonary artery catheter was used. Respiratory failure was severe, and a high concentration of inspired oxygen, high pulmonary end expiratory pressure (PEEP of up to $25 \mathrm{~cm} \mathrm{H}_{2} \mathrm{O}$ ), and recruitment maneuvers guided by CT scan were necessary to maintain adequate oxygenation. The next day after admission to the ICU, a thin blood smear was obtained that revealed $P$. vivax malaria. The diagnosis was confirmed by the Malaria Reference Laboratory (SUCEN, Superintendência de Controle de Endemias do Estado de São Paulo) and there was no microscopic evidence for coinfection with $P$. falciparum. A rapid immunochromatographic test, the Acon Test, (Acon Laboratories, Inc), based on the detection of Histidine Rich Proteins-2 (HRP-2) of P. falciparum, was also negative. Due to the severe clinical picture, she was simultaneously treated with arteemether, primaquine and chloroquine. Parasitemia of the patient became undetectable after $48 \mathrm{hr}$ of treatment. The patient showed progressive improvement, with progressive reductions of the PEEP level and was discharged from the ICU nine days after admission.

Tracheal aspirates were taken when the patient presented respiratory symptoms. The following tests were all negative: rapid test and culture for Influenza $A$ and $B$, direct exam and culture for syncytial respiratory virus, direct immunofluorescence for Cytomegalovirus, and bacterial culture. Four samples from blood cultures were also negative. Cytomegalovirus antigenemia, antibodies against Chlamydia pneumoniae (IgM and $\mathrm{IgG}$ ), Mycoplasma pneumoniae (IgM), and Legionella spp. (IgM) were all negative, and antibodies against dengue virus were negative in two samples obtained during admission and seven days later.

\section{Discussion}

Using the electronic databases of MEDLINE (National Library of Medicine, Bethesda, MD, USA), and manually searching journals published between 1982 and 2003, we found 11 reported cases of vivax malaria with severe pulmonary involvement [6-15]. The main features of these cases, including ours, are presented in Table 1.

There are several clinical manifestations of malaria, and clinical evolution depends on factors of the parasite, the host, and on social and geographic characteristics [16]. The clinical spectrum varies from asymptomatic infection to acute febrile syndrome, severe malaria, and lethal cases. The main clinical manifestation of severe malaria caused by $P$. falciparum includes severe anemia, jaundice, renal failure, cerebral malaria, and ARDS. Sporadically, all of these complications have been reported in vivax malaria [6-15,17,18].

Several pulmonary syndromes in severe vivax malaria have been described, such as acute noncardiogenic pulmonary edema $[8,10]$, ARDS 
Figure 1. Posteroanterior (A) and left lateral (B) chest radiographs of a patient with vivax malaria, showing poorly-defined heterogeneous densification, consistent with alveolar consolidations, predominantly in the posterior and basal regions. There is also a slight blunting of the left costophrenic angle, compatible with pleural effusion.
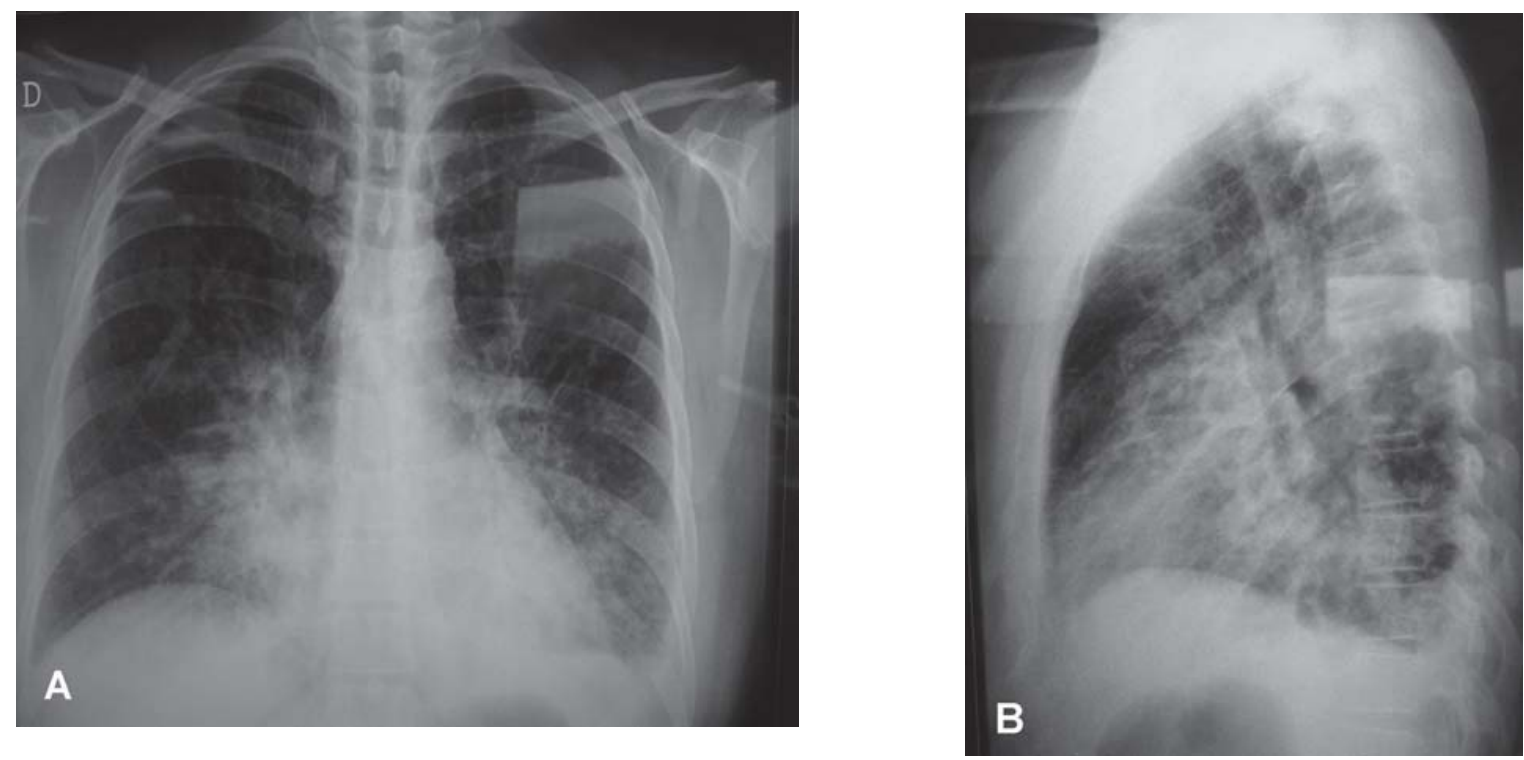

Figure 2. Computerized chest tomography scan of a patient with vivax malaria depicts alveolar consolidations, predominantly distributed in the posterior regions of both lungs (A) and a small bilateral pleural effusion (B).
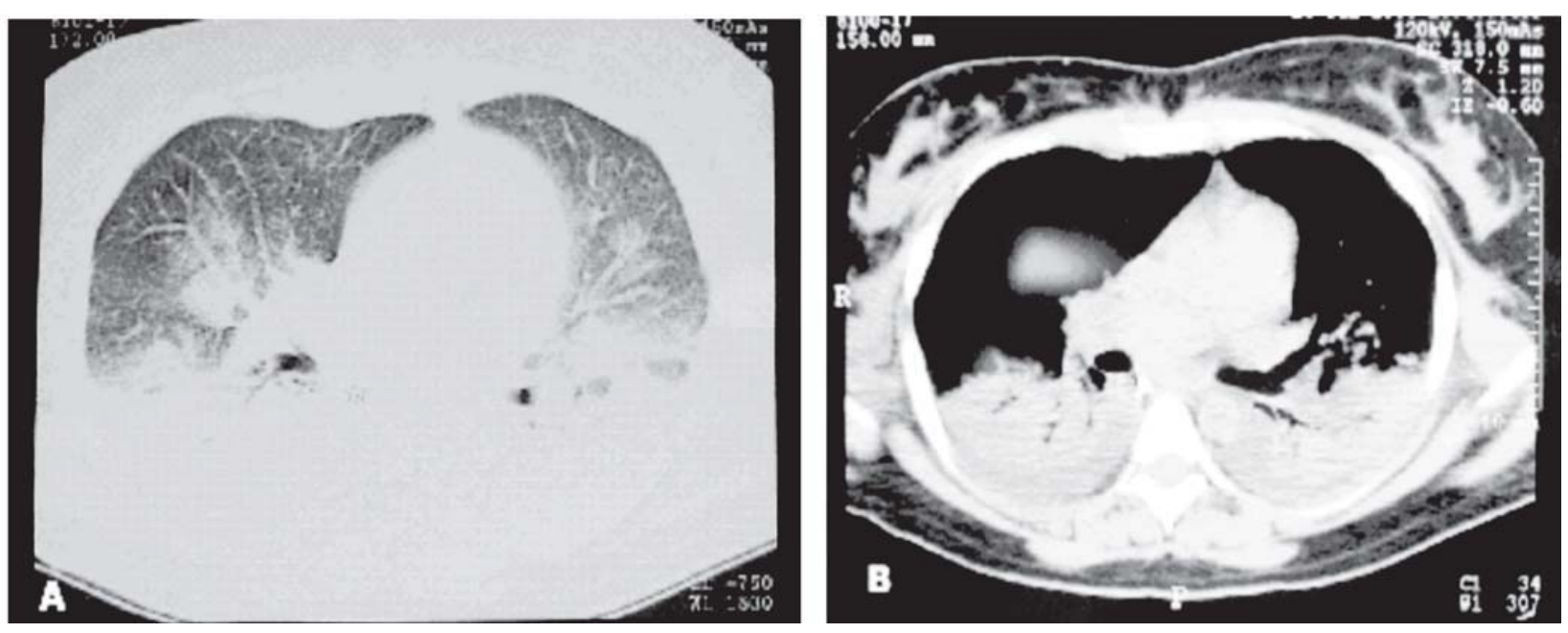


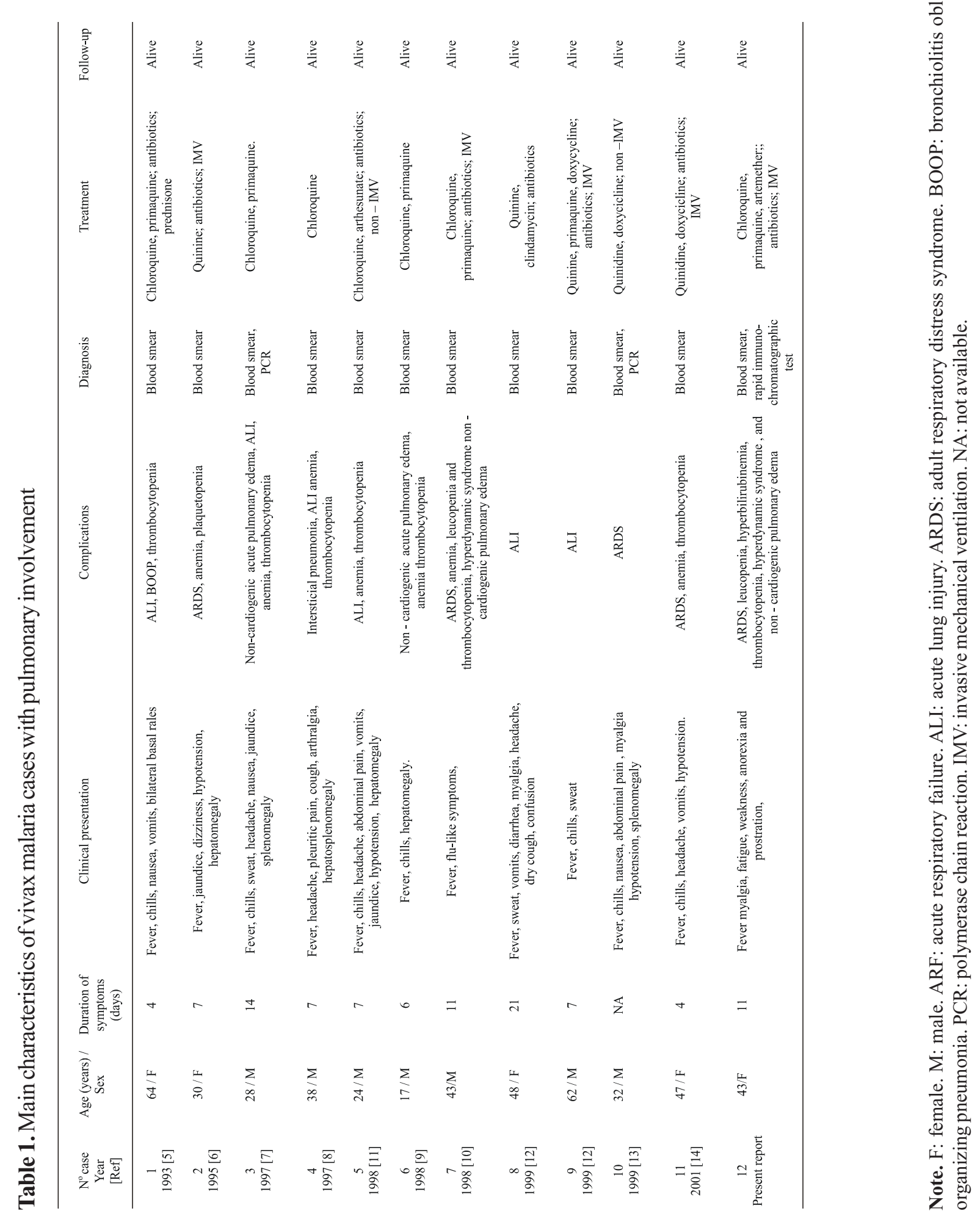


$[7,14,15]$, acute pulmonary injury $[6,8,12]$, and interstitial pneumonia [9]. Respiratory complications of vivax malaria seem to be under-diagnosed. A recent study demonstrated that clinically uncomplicated cases of both falciparum and vivax malaria presented compromised pulmonary function (small airway obstruction, gas exchange alterations, and increased pulmonary phagocytic activity) [22]. Researchers have suggested that an accumulation of pulmonary monocytes may exist, and that an intravascular inflammatory response would follow it, contributing to pulmonary manifestations in malaria [22]. The greater severity and frequency of cases due to falciparum malaria could be explained by a synergy of immune and physical responses, as the parasitized erythrocytes sequester in the pulmonary microcirculation, and by rosetting.

The reviewed cases presented ages between 17 and 64 years. Seven of them reported recent travel to endemic areas $[6,8,12-15]$, showing the importance of obtaining a detailed epidemiological history to consider imported malaria in the differential diagnosis of acute febrile syndrome. Malaria prophylaxis was not used, or was incorrectly used, in most of the cases $[6,8,15]$. Nine patients had no past history for malaria [7,9,11-15]. People living in endemic areas seem to develop some immunity to the parasites, which would partially explain the low frequency of these complications in endemic areas. It is known that nonimmune subjects can present severe clinical manifestations of malarial infection.

Severe pulmonary complications of vivax malaria usually appear from six hours to eight days after the initiation of anti-malarial treatment. These findings are consistent with what is observed in cases of falciparum malaria, and they could correspond to an exacerbation of the post-treatment inflammatory response [22]. Nevertheless, our patient presented severe pulmonary symptoms before the initiation of anti-malarial treatment, which was previously related in only one case, characterized as interstitial pneumonia [9]. These two cases suggest that severe pulmonary involvement is not necessarily a consequence of an inflammatory response produced after anti-malarial treatment.
It is difficult to completely rule out the possibility of co-infection with $P$. falciparum by using conventional parasitological diagnostic tools, which could justify, in some cases, initiation of treatment for both $P$. falciparum and $P$. vivax $[7,12,14,15]$. The level of parasitemia is variable in cases of severe pulmonary involvement. Two patients presented parasitemia counts higher than $2 \%[8,14]$, and one other case was defined as "heavy" [7]. On the other hand, four patients had levels of parasitemia that were considered low: three less than $2 \%[13,15]$, and one case with 1,784 trophozoites $/ \mathrm{mm}^{3}$ [10]. In some cases, worsening of the clinical picture was seen after reduction of parasite counts, reinforcing the possibility of a mediated inflammatory response [22].

Several new technologies for malaria diagnosis have been developed. In two cases in this series, confirmation was made through molecular detection of $P$. vivax DNA by polymerase chain reaction (PCR) $[8,14]$. In addition, immunochromatographic assays allow rapid diagnosis of $P$. falciparum malaria. Preferred targeted antigens are HRP-2 from $P$. falciparum, and parasite-specific lactate dehydrogenase. Since HRP-2 is expressed only by $P$. falciparum, its clinical usefulness for malaria diagnosis is limited, because many cases of nonfalciparum malaria may be misdiagnosed as malaria negative [23]. However, in cases of severe malaria due to $P$. vivax, a rapid immunochromatographic test could be useful to reduce the possibility of $P$. falciparum malaria or mixed malaria.

Due to the clinical possibility of bacterial pneumonia, most of the patients received antibiotics $[6,7,11-13,15]$. Anstey et al., raised the hypothesis that the widespread use of chloroquine and doxycycline in malaria-endemic areas could be attenuating or diminishing the number of severe cases of vivax malaria because of the antiinflammatory properties of these drugs [22]. The possible role of an inflammatory mechanism in pulmonary damage by vivax malaria suggests a potential benefit for the use of corticotherapy. However, there is no evidence that supports this therapeutic approach. The use of corticosteroids was reported in only one case diagnosed as bronchiolitis obliterans organizing pneumonia (BOOP), secondary to malaria [6]. As was 
evident in all cases of severe pulmonary involvement due to vivax malaria, including ours, the use of antimalarial treatment with respiratory support is associated with a good outcome.

In conclusion, vivax malaria should always be considered in the differential diagnosis of patients with consistent epidemiological history, acute febrile syndrome and severe respiratory symptoms before anti-malarial treatment.

\section{Acknowledgments}

We are grateful to George Schulte and Connie Nadler for their assistance in the preparation of the manuscript, and to Marcelo Funari for his radiological descriptions. José E. Vidal was supported by the Coordenação de Aperfeiçoamento de Pessoal de Nível Superior (CAPES) do Ministério da Educação do Brazil (001/0700/000186/2003).

\section{References}

1. Bremen J. The ears of the hippopotamus: manifestations, determinants, and estimates of the malaria burden. Am J Trop Med Hyg 2001;64(Suppl 1-2):1-11.

2. Fairhurst R.M., Wellems T.E. Plasmodium species (Malaria). In: Mandell G.L., Bennett J.E., Dolin R., [eds.] Mandell, Douglas, and Bennett's. Principles and Practice of Infectious Diseases. Sixth edition. Philadelphia: Elsevier Churchill Livingstone, 2005:3121-44.

3. Greenwood B., Mutabingwa T. Malaria in 2002. Nature 2002;415:670-2.

4. Maitland K., Bejon P., Newton C.R. Malaria. Curr Opin Infect Dis 2003; 16:389-95.

5. World Health Organization. Severe falciparum malaria. Trans R Soc Trop Med Hyg 2000; 94( Suppl 1):1-90.

6. Yale S., Adlakha A., Sebo T.J., Ryu J.H. Bronchiolitis obliterans organizing pneumonia caused by Plasmodium vivax malaria. Chest 1993; 104:1294-6.

7. Islam N., Qamruddink K. Unusual complications in benign tertian malaria. Trop Geogr Med 1995;47:141-3.

8. Torres J.R., Perez H., Postigo M.M., Silva J.R. Acute noncardiogenic lung injury in benign tertian malaria. Lancet 1997;350:31-2.
9. Munteis E., Melliborsky L., Marques M.A., et al. Pulmonary involvement in a case of Plasmodium vivax malaria [letter]. Chest 1997;111:834-5.

10. Pukrittayakamee S., Chantra A., Vanijanonta S., White N.J. Pulmonary edema in vivax malaria. Trans R Soc Trop Med Hyg 1998;92:421-2.

11. Perren A., Beretta F., Schubarth P. ARDS in Plasmodium vivax malaria. Schweiz Med Wochenschr 1998; $128: 1020-3$.

12. Kalmar E.M., Cassetari V., Machado F.R., et al. Malaria grave em paciente com infecção por Plasmodium vivax: relato de caso. Rev Soc Bras Med Trop 1998;31(Suppl 1): $\mathrm{P} 090$.

13. Curlin M.E., Barat L.M., Walsh D.K., Granger D.L. Noncardiogenic pulmonary edema during vivax malaria. Clin Infect Dis 1999;28:1166-7.

14. Carlini M.E., White A.C., Atmar R.L. Vivax malaria complicated by adult respiratory distress syndrome. Clin Infect Dis 1999;28:1182-3.

15. Tanios M.A., Kogelman L., McGovern B., Hassoun P. Acute respiratory distress syndrome complicating Plasmodium vivax malaria. Crit Care Med 2001;29:665-7.

16. Miller L.H., Baruch D.I., Marsh K., Doumbo O.K. The pathogenic basis of malaria. Nature 2002;415:673-9.

17. Oh M.D., Shin H., Shin D., et al. Clinical features of vivax malaria. Am J Trop Med Hyg 2001;65:143-6.

18. Beg M.A., Khan R., Baig S.M., et al. Cerebral involvement in benign tertian malaria. Am J Trop Med Hyg 2002; $67: 230-2$.

19. Chotivanich K., Udomsagpetch R., Simpson J.A., et al. Parasite multiplication potential and the severity of falciparum malaria. J Infect Dis 2000;181:1206-9.

20. Chen Q., Schilichtherle M., Wahlgren M. Molecular aspects of severe malaria. Clin Microbiol Rev 2000; $13: 439-50$.

21. Clark I.A., Cowden W.B. Why is the pathology of falciparum worse than that of vivax malaria? Parasitol Today 1999; 15:458-61.

22. Anstey N.M., Jacups S.P., Cain T., et al. Pulmonary manifestations of uncomplicated falciparum and vivax malaria: caught, small airways obstruction, impaired gas transfer, and increased pulmonary phagocytic activity. J Infect Dis 2002;185:1326-34.

23. Moody A. Rapid diagnostic test for malaria. Clin Microbiol Rev 2002; 15:66-78. 